\title{
Desarrollo organizacional en la gestión municipal
}

\author{
Nino Alvarez Rios \\ abogadoninoalvarezrios@outlook.com \\ Escuela de posgrado \\ Universidad César Vallejo \\ ORCID: 0000-0001-8678-5424 \\ José Manuel Delgado Bardales \\ jmdelgadob@ucvvirtual.edu.pe \\ Escuela de posgrado \\ Universidad César Vallejo \\ ORCID:0000-0001-6574-2759 \\ Scopus autor ID: 24070333700 \\ Código Renacyt: P0050554
}

\section{RESUMEN}

La presente investigación tuvo como objetivo, Caracterizar el desarrollo organizacional de la gestión en las municipalidades, la investigación fue cualitativa de diseño revisión sistemática, se realizó el análisis de 10 artículos científicos relacionados a la variable de estudio. Se encontró que el 50\% de los artículos científicos se encuentran enfocados a desarrollo de competencias y habilidades de los trabajadores municipales a través de capacitaciones, 30\% se encuentra enfocado al uso de la TIC, para lograr la gestión y $20 \%$ menciona el uso de la coeficiencia y metodologías aplicadas a al mapeo de objetivos institucionales. La gestión de las municipalidades son deficientes en Asia, Europa y África, las autoridades y directivos de los municipios deben tomar en cuenta diferentes metodologías para el desarrollo organizacional. Además, en el desarrollo de futuras investigaciones tomar variables y dimensiones de gestión que permitan escudriñar con mayor énfasis en la problemática que aquejan los gobiernos locales.

Palabras clave: Desarrollo organizacional, gestión, municipalidad. 


\title{
Organizational development in municipal management
}

\begin{abstract}
The objective of this research was to characterize the organizational development of management in the municipalities, the research was qualitative with a systematic review design, the analysis of 10 scientific articles related to the study variable was carried out. It was found that $50 \%$ of the scientific articles are focused on the development of skills and abilities of municipal workers through training, $30 \%$ are focused on the use of ICT, to achieve management and $20 \%$ mention the use of the coefficients and methodologies applied to the mapping of institutional objectives. The management of municipalities is deficient in Asia, Europe and Africa, authorities and managers of municipalities must take into account different methodologies for organizational development. In addition, in the development of future research, take variables and management dimensions that allow scrutinizing with greater emphasis on the problems faced by local governments.
\end{abstract}

Keywords: Organizational development, management, municipality.

Artículo recibido: 03 nov. 2020

Aceptado para publicación: 07 dic. 2020

Correspondencia bogadoninoalvarezrios@outlook.com

Conflictos de Interés: Ninguna que declarar 


\section{INTRODUCCIÓN}

Es de público conocimiento que, la gestión municipal es el órgano de más alto nivel técnico administrativo, cuya función es de conducir y dirigir la gestión financiera del gobierno municipal en su conjunto, así como prestar servicios públicos y promover inversiones de obras de su competencia. Por tal motivo, el alcalde es quien se encarga de ejecutar proyectos que están inmersos en el Plan de Desarrollo Concertado, el presupuesto participativo y las políticas de gestión, etc. En estos últimos años, el tema de la gestión administrativa en las municipalidades está dando mucho que hablar, debido a la falta de liderazgo y motivación que demuestran los alcaldes y toda su plana administrativa de turno. Esto se debe a diversos factores, siendo el principal la falta de habilidades gerenciales para dirigir con equidad los recursos humanos y materiales de su institución.

Cabe mencionar que, en los países europeos y de América del Norte, la realidad es otra, los gobiernos municipales, los alcaldes han establecido una corriente la cual se transformó en cultura de innovación como parte prioritaria dentro de su plan de gobierno. En Latinoamérica, se evidencia la falta de habilidades gerenciales de los alcaldes para solucionar los problemas de la ciudanía y como también una pésima atención a los administrados. Según concepción de, (Tirado, 2014. p.54), la gestión administrativa son actividades que se aplican de manera coordinada el esfuerzo de un equipo de trabajadores, es decir, la forma como se busca de llegar a las metas y objetivos de manera conjunta pasando por el proceso de planeación, organización, dirección y control (p.70). En cambio, para, Latinobarometro, (2017), el 53\% de la población latinoamericana opina que es poco probable que los gobiernos municipales den respuesta a los principales problemas de la sociedad, lo que indica una demanda ciudadana por la consolidación de gobiernos locales capaces de hacer frente a sus necesidades (p.70).

En el Perú, nuestros gobiernos municipales atraviesan una crisis en cuanto a la gestión administrativa, los alcaldes que fueron elegidos por un pedido determinado, adolecen de liderazgo, no se evidencia el trabajo en equipo, falta de compromiso, existes muchos conflictos, maltrato a los administrados; ocasionando que los proyectos de inversión pública se vean afectados, lo que demuestra, que, la mayoría de los burgomaestres carezcan de habilidades gerenciales para dirigir una organización (Urbina, 2017. p. 35).

En tal sentido la Municipalidad distrital de Morales, pertenece a la provincia y región San Martín, donde el alcalde ostenta la máxima autoridad cuya función es administrar los ingresos y egresos económicos; además de realizar obras que beneficien a la comunidad de su jurisdicción y buscar el desarrollo social, económico sostenible. 
Lo cual lamentablemente, en los últimos tiempos, la gestión del alcalde y su plana administrativa elegida, vienen siendo cuestionados severamente por irregularidades en su gestión; desde un inicio los conflictos por las relaciones interpersonales se vieron afectadas por falta de comunicación y liderazgo, donde los conflictos fueron del dominio público que ponían de manifiesto la incapacidad para tomar decisiones acertadas y la imposibilidad para solucionar los problemas de manera inmediata de la población; por lo que se deduce que los funcionarios municipales carecen de habilidades gerenciales para administrar al capital humano y material de su comunidad. (Miranda, 2016. p. 34), es de suma urgencia urge elaborar una propuesta metodológica que contribuirá a desarrollar las habilidades y una buena praxis gerencial de los que administran la gestión pública. Por lo cual se propone como problema general, la siguiente interrogante; ¿Cuáles son las características del desarrollo organizacional en la gestión municipal? Asimismo, como objetivo de la investigación, Caracterizar el desarrollo organizacional de la gestión en las municipalidades.

\section{ESTRATEGIAS METODOLÓGICAS O MATERIALES Y MÉTODOS}

El tipo es cualitativo y el diseño de estudio es una revisión sistemática, las revisiones sistemáticas son investigaciones científicas en las cuales la unidad de análisis son los estudios originales primarios, constituyen una herramienta esencial para sintetizar la información científica disponible, incrementar la validez de las conclusiones de estudios individuales e identificar áreas de incertidumbre donde sea necesario realizar investigación. (Muñoz, 2007. p. 364)

La recolección de datos se realizó a través de la revisión bibliográfica de artículos de investigaciones tanto nacionales como internacionales que tuvieron como tema principal el consumo y su influencia en la vida sostenible teniendo en cuenta la crisis económica; de todos los artículos que se encontraron, se incluyeron los más importantes según nivel de evidencia y se excluyeron los menos relevantes. Se estableció la búsqueda siempre y cuando se tuvo acceso al texto completo del artículo científico.

El análisis de la revisión sistemática evaluando cada uno de los artículos para una comparación de los puntos o características en las cuales concuerda y los puntos en los que existe discrepancia entre artículos nacionales e internacionales. Además, de acuerdo a criterios técnicos pre establecidos, se realizó una evaluación crítica e intensiva de cada artículo, a partir de ello, se determinó la calidad de la evidencia y la fuerza de recomendación para cada artículo. 


\section{RESULTADOS Y DISCUSIÓN}

\section{Resultados}

Tablas 1: Estudios revisados sobre el desarrollo organizacional y la gestión en las municipalidades

\begin{tabular}{|c|c|c|c|c|c|c|}
\hline \multicolumn{7}{|c|}{ DATOS DE LA PUBLICACIÓN } \\
\hline 1. Autor & Año & \multicolumn{3}{|c|}{ Nombre de la Investigación } & $\begin{array}{c}\text { Revista donde se ubica la } \\
\text { Publicación }\end{array}$ & Volumen y Numero \\
\hline $\begin{array}{l}\text { Al-Bataineh, O. H., } \\
\text { Ibrahim, R. B. M., \& } \\
\text { Fadzil, A. F. M. }\end{array}$ & 2019 & \multicolumn{3}{|c|}{$\begin{array}{l}\text { The Effects of Motivation, Empowerment } \\
\text { and Organizational culture on Organizational } \\
\text { Commitment of Municipalities in Jordan }\end{array}$} & $\begin{array}{c}\text { http://hrmars.com/index.php/journals/ } \\
\text { papers/IJARBSS/v9-i9/6272 } \\
\text { Jordania }\end{array}$ & $\begin{array}{l}\text { International Journal of } \\
\text { Academic Research in } \\
\text { Business and Social Sciences } \\
2019 \text { vol: } 9 \text { (9) pp: } 101-117\end{array}$ \\
\hline \multicolumn{7}{|c|}{ CONTENIDO DE LA PUBLICACIÓN } \\
\hline $\begin{array}{l}\text { Tipo y Diseño de } \\
\text { Investigación }\end{array}$ & $\begin{array}{r}\text { Poblaci } \\
\text { Muest }\end{array}$ & & Instrumentos & Aspectos éticos & Resultados & Conclusión \\
\hline Correlación & $\begin{array}{r}\text { este es } \\
\text { utilizó un } \\
\text { diecioch } \\
\text { artículo } \\
\text { medi } \\
\text { motiva } \\
\text { quince } \\
\text { element } \\
\text { medi } \\
\text { Empoder }\end{array}$ & $\begin{array}{l}\text { dio } \\
\text { otal de } \\
(18) \\
\text { para } \\
\text { la } \\
\text { ón y } \\
\text { (15) } \\
\text { s para } \\
\text { el } \\
\text { miento }\end{array}$ & $\begin{array}{l}\text { este estudio } \\
\text { utilizó un total } \\
\text { de dieciocho } \\
\text { (18) artículos } \\
\text { para } \\
\text { medir la } \\
\text { motivación ) }\end{array}$ & $\begin{array}{l}\text { Consentimiento } \\
\text { informado. }\end{array}$ & $\begin{array}{l}\text { Los resultados mostraron ese coeficiente de } \\
\text { trayectoria, y el valor } \mathrm{T} \text { y }(\beta=0.161, \mathrm{t}= \\
1.784) \text {. Por lo tanto, H1 es apoyado y esta } \\
\text { línea de resultados con Van der Voet, Steijn } \\
\text { et al. (2017) y Laschinger, Read et Alabama. } \\
\text { (2016) Además, los resultados indicaron que } \\
\text { el empoderamiento se asocia positivamente } \\
\text { con compromiso organizacional ( } \beta=0.476 \text {; } \\
\mathrm{t}=7.321) \text {, por lo tanto, H2 es compatible y } \\
\text { se alinea con Han, Seo } \\
\text { et al. (2016) Además, H3 propuso que la } \\
\text { relación entre cultura organizacional }\end{array}$ & $\begin{array}{l}\text { Los hallazgos se han } \\
\text { esforzado empíricamente para } \\
\text { guiar a los responsables } \\
\text { políticos en Jordania y } \\
\text { futuros investigadores verán } \\
\text { el papel de la motivación } \\
\text { examinada, el } \\
\text { empoderamiento } \\
\text { y cultura organizacional para } \\
\text { obtener un mejor compromiso } \\
\text { organizacional, especialmente } \\
\text { en el países en desarrollo. }\end{array}$ \\
\hline
\end{tabular}




\begin{tabular}{|c|c|c|c|c|c|c|}
\hline \multicolumn{2}{|c|}{ 2. Autor } & Año & Nombre de la Investig & ción & Revista donde se ubica la Publicación & Volumen y Numero \\
\hline \multicolumn{7}{|c|}{ CONTENIDO DE LA PUBLICACIÓN } \\
\hline $\begin{array}{l}\text { Tipo y Diseño de } \\
\text { Investigación }\end{array}$ & $\begin{array}{l}\text { Población } \\
\text { y Muestra }\end{array}$ & & Instrumentos & $\begin{array}{c}\text { Aspectos } \\
\text { éticos }\end{array}$ & Resultados & Conclusión \\
\hline
\end{tabular}

\begin{tabular}{ccccc}
\hline 3. Autor & Año & Nombre de la Investigación & Revista donde se ubica la Publicación & Volumen y Numero \\
\hline \multirow{2}{*}{ De Gier, J. } & \multirow{2}{*}{2017} & $\begin{array}{l}\text { IT Governance of Dutch } \\
\text { Municipalities and Digital } \\
\text { Information Management }\end{array}$ & https://www.tandfonline.com/doi/full/10.108 & Volume 23, 2018 - \\
& & New Review of Information Networking & Issue $1-2$ & \\
\hline
\end{tabular}




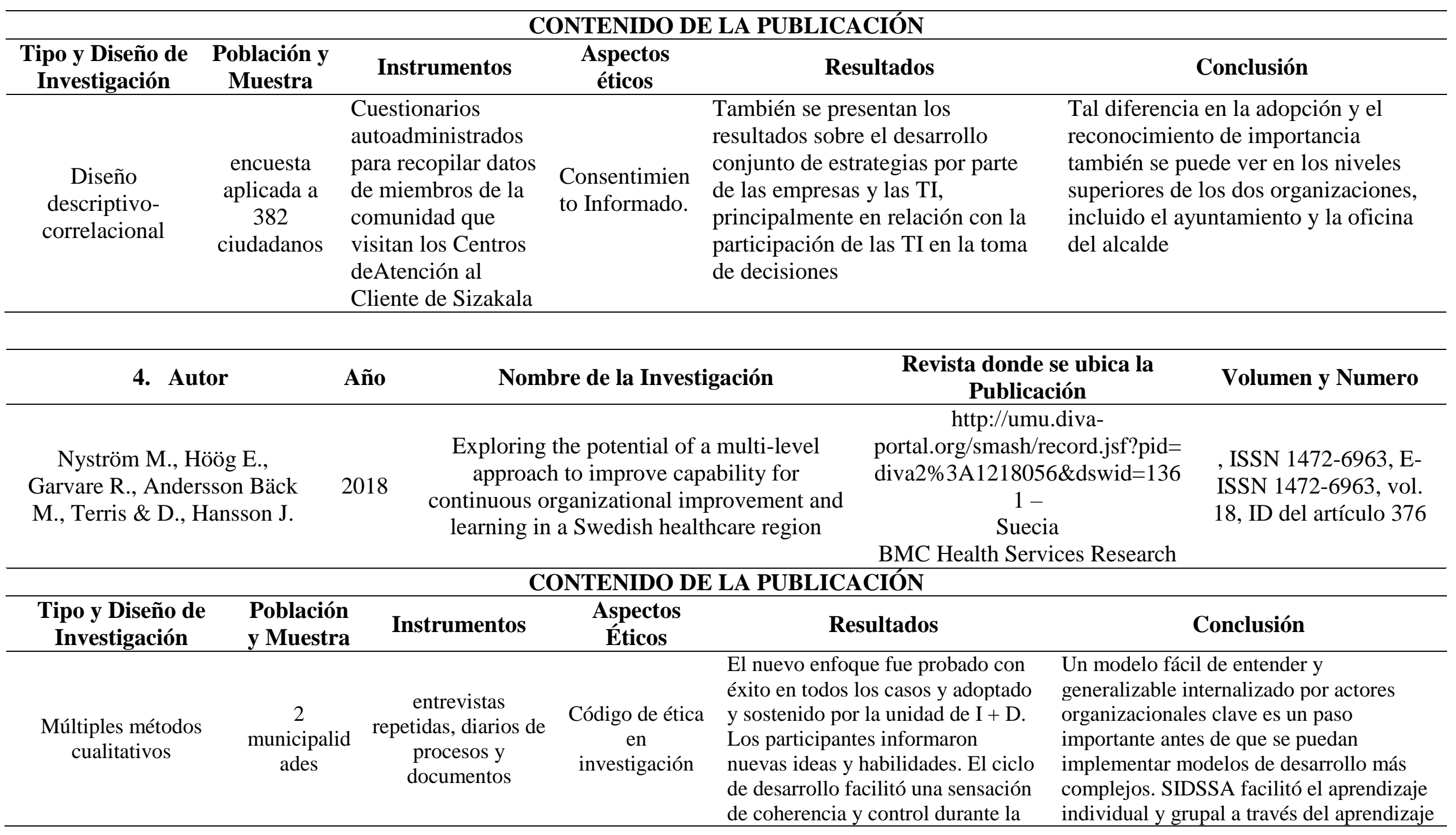


incertidumbre, mejoró la

planificación y el análisis de problemas, mejoró el mapeo del contexto y las condiciones, y apoyó la resolución de problemas tanto a nivel individual como a nivel de unidad. La visión a nivel de sistemas y el enfoque estructurado ayudaron a los participantes a explicar, motivar e implementar iniciativas de cambio,

especialmente después de trabajar de manera más sistemática con el

mapeo, el análisis y el establecimiento de objetivos. en acción y apoyó vistas a nivel de sistemas y enfoques estructurados en múltiples niveles organizacionales. Se facilitó la participación activa de diversas funciones y niveles organizativos en el proceso de aprendizaje. Sin embargo, el período de tiempo era demasiado corto para probar completamente todos los aspectos del enfoque, específicamente para llegar más allá de los gerentes involucrados al personal de primera línea y los pacientes.

\begin{tabular}{|c|c|c|c|c|c|c|c|}
\hline \multicolumn{2}{|c|}{ 5. Autor } & Año & \multicolumn{3}{|c|}{ Nombre de la Investigación } & Revista donde se ubica la Publicación & Volumen y Numero \\
\hline \multicolumn{2}{|c|}{$\begin{array}{l}\text { Díaz-Villavicencio G., } \\
\text { Didonet S., \& } \\
\text { Dodd A. }\end{array}$} & 2017 & \multicolumn{3}{|c|}{$\begin{array}{l}\text { Influencing factors of eco-efficient } \\
\text { urban waste management: Evidence } \\
\text { from Spanish municipalities }\end{array}$} & $\begin{array}{l}\text { https://www.sciencedirect.com/science/art } \\
\text { icle/pii/S0959652617314920 } \\
\text { España, Europa }\end{array}$ & $\begin{array}{l}\text { Journal of Cleaner } \\
\text { Production } \\
\text { 2017 vol: } 164 \mathrm{pp:} \\
\text { 1486-1496 }\end{array}$ \\
\hline \multicolumn{8}{|c|}{ CONTENIDO DE LA PUBLICACIÓN } \\
\hline $\begin{array}{c}\text { Tipo y Diseño } \\
\text { de Investigación }\end{array}$ & $\begin{array}{c}\text { Población y } \\
\text { Muestra }\end{array}$ & Ins & Imentos & $\begin{array}{c}\text { Aspectos } \\
\text { éticos }\end{array}$ & & Resultados & Conclusión \\
\hline Descriptivo & $\begin{array}{l}143 \\
\text { municipios } \\
\text { españoles. }\end{array}$ & $\begin{array}{l}\text { Enc } \\
\text { cue }\end{array}$ & $\begin{array}{l}\text { sta- } \\
\text { nario }\end{array}$ & $\begin{array}{l}\text { Código de } \\
\text { ética en } \\
\text { investigación }\end{array}$ & $\begin{array}{l}\text { El resultad } \\
\text { la empatía, } \\
\text { variables p } \\
\text { del servicio } \\
\text { fiabilidad, } \\
\text { que hacen }\end{array}$ & $\begin{array}{l}\text { le este estudio indica que, excepto } \\
\text { dos } \\
\text { dictoras o dimensiones de calidad } \\
\text { angibilidad, } \\
\text { pacidad de respuesta y seguridad) } \\
\text { estadística }\end{array}$ & $\begin{array}{l}\text { dio muestra que una } \\
\text { ración local no necesita } \\
\text { alto nivel económico o } \\
\text { y poblada para alcanzar } \\
\text { nivel de eco eficiencia. } \\
\text { dinero lo que causa un } \\
\text { de eco eficiencia, }\end{array}$ \\
\hline
\end{tabular}


contribución significativa en influencia mejorando significativamente la combinada la satisfacción de clientes. Por otro organización

lado, la empatía no tiene un efecto significativo.

\begin{tabular}{|c|c|c|c|c|c|c|}
\hline 6. Autor & Año & \multicolumn{2}{|c|}{ Nombre de la Investigación } & \multicolumn{3}{|c|}{ Revista donde se ubica la Publicación $\quad$ Volumen Y Numero } \\
\hline Manuere et al., & 2017 & \multicolumn{2}{|c|}{$\begin{array}{l}\text { Human Capital Development } \\
\text { Programmes and their Effect on } \\
\text { the Job Satisfaction of Workers in } \\
\text { Zimbabwe Urban Municipalities: } \\
\text { The Case of Chinhoyi }\end{array}$} & $\begin{array}{r}\text { International Journal o } \\
\text { Research in Business and } \\
\text { https://econpapers.repec.o } \\
\text { rbs/v_3a7_3ay_3a2017_3 } \\
\text { a874-889.ht } \\
\text { Zimbabwe, Áf }\end{array}$ & $\begin{array}{l}\text { Academic } \\
\text { ocial Sciences } \\
\text { g/article/hurija } \\
\text { i_3a12_3ap_3 } \\
\text { ica }\end{array}$ & $\begin{array}{l}\text { vol. } 7 \text {, issue } 12,874- \\
889\end{array}$ \\
\hline \multicolumn{7}{|c|}{ CONTENIDO DE LA PUBLICACIÓN } \\
\hline $\begin{array}{c}\text { Aplicado, } \\
\text { Cuantitativo, } \\
\text { correlacional }\end{array}$ & $\begin{array}{l}\text { El tamaño de } \\
\text { la muestra de } \\
\text { los } \\
\text { participantes } \\
\text { en la } \\
\text { investigación } \\
\text { fue de } 30\end{array}$ & $\begin{array}{l}\text { El cuestionario } \\
\text { se caracterizó } \\
\text { por ítems de } \\
\text { escala Likert } \\
\text { susceptible de } \\
\text { análisis } \\
\text { estadístico. }\end{array}$ & $\begin{array}{c}\text { Código de ética } \\
\text { en } \\
\text { investigación }\end{array}$ & $\begin{array}{l}\text { Se muestra que la hipótesis } \\
\text { nula no puede ser aceptada. El } \\
\text { valor significativo (.043) que } \\
\text { es } \\
\text { relacionado con el valor } \\
\text { calculado t }(2,63) \text { es menor } \\
\text { que el valor crítico del } 5 \% \text {. } \\
\text { Esto significa que hay una } \\
\text { relación positiva entre } \\
\text { capacitación y desarrollo y } \\
\text { satisfacción laboral de los } \\
\text { empleados. }\end{array}$ & $\begin{array}{l}\text { Los resultados } \\
\text { que existe una } \\
\text { el entrenamien } \\
\text { satisfacción la } \\
\text { gestión munici }\end{array}$ & $\begin{array}{l}\text { del estudio revelan } \\
\text { relación positiva entre } \\
\text { to y desarrollo y } \\
\text { boral, mejorando la } \\
\text { pal. }\end{array}$ \\
\hline
\end{tabular}

\begin{tabular}{|c|c|c|c|c|}
\hline 7. Autor & Año & Nombre de la Investigación & Revista donde se ubica la Publicación & Volumen Y Numero \\
\hline $\begin{array}{l}\text { Jašarević S., Miličević D., } \\
\text { Brdarević S., \& Lemeš S. }\end{array}$ & 2018 & $\begin{array}{l}\text { Measurement of organizational } \\
\text { culture in public administration: }\end{array}$ & $\begin{array}{l}\text { International Journal for Quality } \\
\text { Research }\end{array}$ & \\
\hline
\end{tabular}

Ciencia Latina Revista Científica Multidisciplinar, Ciudad de México, México.

ISSN 2707-2207 / ISSN 2707-2215 (en línea), julio-diciembre, 2020, Volumen 4, Número 2.

https://doi.org/10.37811/cl_rcm.v4i2.133 p. 963 


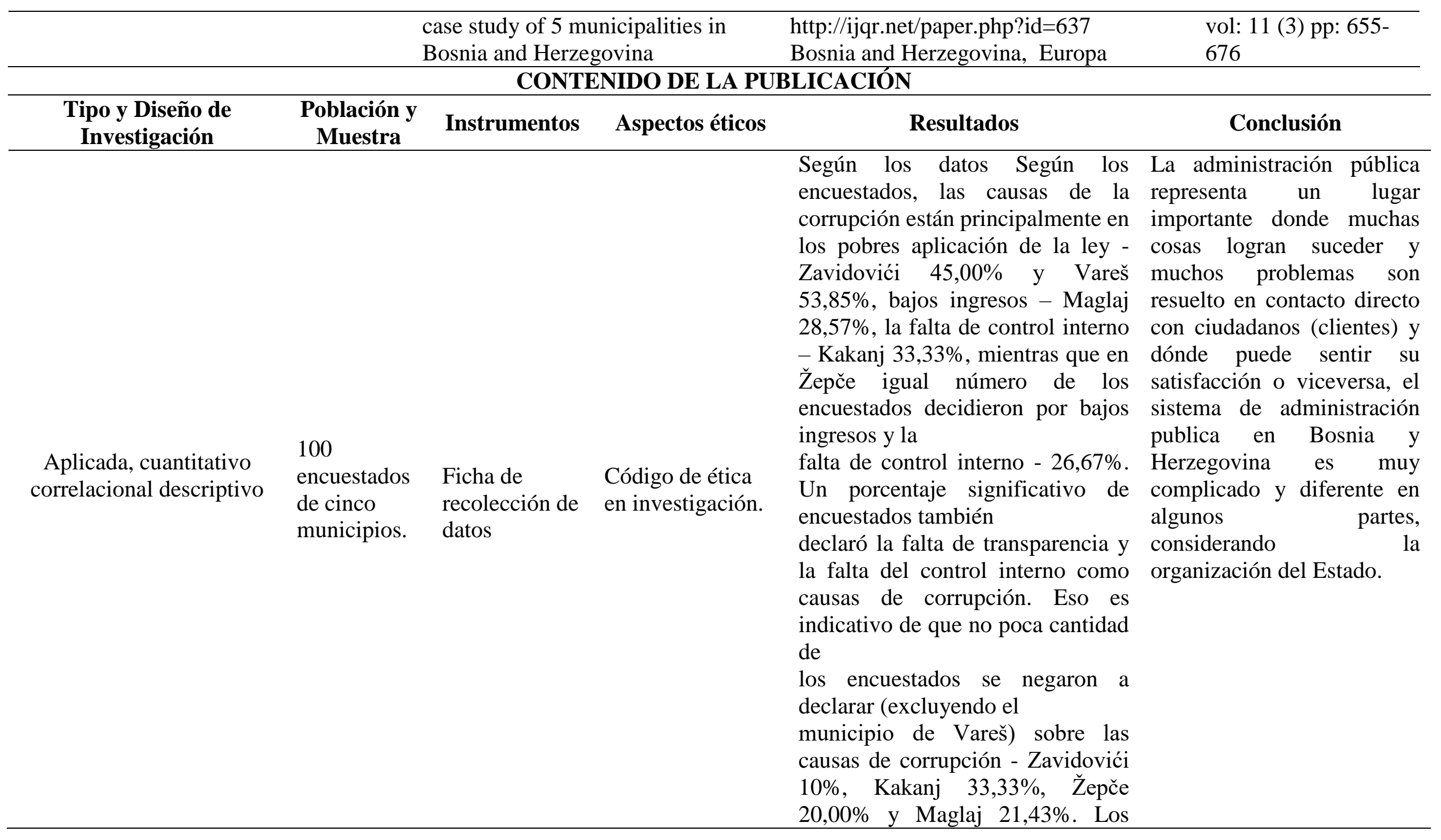


resultados indican la existencia de

desviaciones

comportamiento, falta de reglas de

conducta, disfunción

organizacional y pobre Motivación

de los empleados. (Tabla 16)

\begin{tabular}{|c|c|c|c|c|c|c|c|}
\hline \multicolumn{2}{|c|}{ 8. Autor } & Año & \multicolumn{2}{|c|}{$\begin{array}{l}\text { Nombre de la } \\
\text { Investigación }\end{array}$} & \multicolumn{2}{|c|}{ Revista donde se ubica la Publicación } & Volumen y Numero \\
\hline $\begin{array}{r}\text { Lo Salem Deeb B. El } \\
\text { Al-Habil W }\end{array}$ & Shorafa Y. & 2019 & \multicolumn{2}{|c|}{$\begin{array}{l}\text { The Effect of Some } \\
\text { Organizational Factors on } \\
\text { the Knowledge } \\
\text { Management } \\
\text { Implementation at the } \\
\text { Municipality of Gaza }\end{array}$} & \multicolumn{2}{|c|}{$\begin{array}{c}\text { https://www.semanticscholar.org/paper/The-Effect- } \\
\text { of-Some-Organizational-Factors-on-the-at-Al-Habil- } \\
\text { El- } \\
\text { Shorafa/b8a5199d4e4294e2fe1fb713bcf4fb674ce4b6 } \\
60 \text { IUG Journal of Economics and Business Studies, } \\
\text { Gaza, Palestina }\end{array}$} & vol: 24 (1) pp: $1-16$ \\
\hline \multicolumn{8}{|c|}{ CONTENIDO DE LA PUBLICACIÓN } \\
\hline $\begin{array}{l}\text { Tipo y Diseño de } \\
\text { Investigación }\end{array}$ & $\begin{array}{l}\text { Población } \\
\text { y Muestra }\end{array}$ & \multicolumn{2}{|c|}{ Instrumentos } & $\begin{array}{c}\text { Aspectos } \\
\text { éticos }\end{array}$ & \multicolumn{3}{|c|}{ Conclusión } \\
\hline Estudio empírico & $\begin{array}{c}240 \\
\text { empleados }\end{array}$ & $\begin{array}{r}\text { des } \\
\text { pr } \\
\text { cues } \\
10 \\
\text { dis } \\
\text { perso } \\
\text { una } m\end{array}$ & $\begin{array}{l}\text { rrolló y } \\
\text { bó un } \\
\text { onario y } \\
\text { go lo } \\
\text { ribuyó } \\
\text { almente a } \\
\text { lestra que } \\
\text { onsta }\end{array}$ & $\begin{array}{l}\text { Código de ética } \\
\text { en investigaciór }\end{array}$ & $\begin{array}{l}\text { tiene una tasa de respuesta del 94\%. El estudio } \\
\text { encuentra que } 67 \text {. El } 26 \% \text { del personal de la } \\
\text { Municipalidad de Gaza estuvo de acuerdo en } \\
\text { que existe un efecto estadísticamente } \\
\text { significativo de la implementación de la } \\
\text { Gestión del Conocimiento en el desarrollo del } \\
\text { desempeño administrativo y del servicio, esto } \\
\text { revela que la Gestión del Conocimiento es una } \\
\text { herramienta efectiva y afecta fuertemente el } \\
\text { desempeño }\end{array}$ & $\begin{array}{l}\text { El estudic } \\
\text { centro cer } \\
\text { la creació } \\
\text { intercamb } \\
\text { experime } \\
\text { habilidad } \\
\text { amplio co } \\
\text { normas, } \\
\text { Municipi }\end{array}$ & $\begin{array}{l}\text { ecomienda iniciar un nuevo } \\
\text { al como infraestructura para } \\
\text { de conocimiento y el } \\
\text { con un equipo } \\
\text { do que no solo tenga } \\
\text { de gestión, sino también un } \\
\text { cimiento de la estrategia, las } \\
\text { servicios y las prácticas del } \\
\text { le Gaza. }\end{array}$ \\
\hline
\end{tabular}

Ciencia Latina Revista Científica Multidisciplinar, Ciudad de México, México. ISSN 2707-2207 / ISSN 2707-2215 (en línea), julio-diciembre, 2020, Volumen 4, Número 2. https://doi.org/10.37811/cl_rcm.v4i2.133 p. 965 


\section{Autor Año Nombre de la Investigación}

Dadashi Jokandan A. Fattahi M. \& Ghane $\mathrm{S}$.

Examining the Role of Information and Communication

2015 Technology in the Organizational Structure of the QaemShahr Municipality

\begin{tabular}{|c|c|c|c|c|c|}
\hline & & & \multicolumn{3}{|c|}{ Leadership , Irán } \\
\hline $\begin{array}{l}\text { Tipo y Diseño de } \\
\text { Investigación }\end{array}$ & $\begin{array}{l}\text { Población y } \\
\text { Muestra }\end{array}$ & Instrumentos & $\begin{array}{l}\text { Aspectos } \\
\text { éticos }\end{array}$ & Resultados & Conclusión \\
\hline $\begin{array}{l}\text { Este estudio es una } \\
\text { investigación aplicada que } \\
\text { adopta un método } \\
\text { descriptivo }\end{array}$ & $\begin{array}{c}100 \text { personas del } \\
\text { municipio de } \\
\text { QaemShahr } \\
\text { participaron en el } \\
\text { estudio, de las cuales } \\
80 \text { miembros del } \\
\text { personal fueron } \\
\text { elegidos como } \\
\text { muestra. }\end{array}$ & $\begin{array}{l}\text { Se utilizó un cuestionario } \\
\text { realizado por un } \\
\text { investigador con un } \\
\text { coeficiente alfa de } 0.86 \\
\text { Cronbach y un cuestionario } \\
\text { estándar de Stephen Robins } \\
\text { para evaluar la estructura } \\
\text { organizacional. }\end{array}$ & $\begin{array}{l}\text { Código de ética } \\
\text { en } \\
\text { investigación. }\end{array}$ & $\begin{array}{l}\text { Los resultados indicaron que } \\
\text { las TIC tuvieron un impacto } \\
\text { en la estructura } \\
\text { organizacional y entre las } \\
\text { tres dimensiones de la } \\
\text { estructura, la formalidad tuvo } \\
\text { el más alto nivel de } \\
\text { importancia con las TIC. }\end{array}$ & $\begin{array}{l}\text { No existe una diferencia } \\
\text { significativa en el } \\
\text { conocimiento de las TIC } \\
\text { entre mujeres y hombres, así } \\
\text { como el impacto de las TIC } \\
\text { en la estructura } \\
\text { organizacional a diferentes } \\
\text { niveles en el municipio de } \\
\text { QaemShahr. }\end{array}$ \\
\hline
\end{tabular}

\begin{tabular}{|c|c|c|c|c|c|c|c|}
\hline 10. Autor & Año & \multicolumn{3}{|c|}{ Nombre de la Investigación } & \multicolumn{2}{|c|}{$\begin{array}{c}\text { Revista donde se ubica la } \\
\text { Publicación }\end{array}$} & Volumen y Número \\
\hline $\begin{array}{c}\text { Holagh S., Noubar H., } \\
\text { Bahador B. }\end{array}$ & 2019 & \multicolumn{3}{|c|}{$\begin{array}{l}\text { The Effect of Organizational Structure on } \\
\text { Organizational Creativity and Commitment within the } \\
\text { Iranian Municipalities }\end{array}$} & \multicolumn{2}{|c|}{$\begin{array}{c}\text { https://www.sciencedirect.co } \\
\text { m/science/article/pii/S187704 } \\
\text { 2814059953 } \\
\text { Riga, Latvia } \\
\end{array}$} & $\begin{array}{c}\text { Procedia - Social and } \\
\text { Behavioral Sciences } \\
2014 \text { vol: } 156 \text { pp: } \\
\text { 213-215 } \\
\end{array}$ \\
\hline $\begin{array}{l}\text { Tipo y Diseño de } \\
\text { Investigación }\end{array}$ & $\begin{array}{l}\text { Población y } \\
\text { Muestra }\end{array}$ & Instrumentos & Aspectos éticos & & \multicolumn{3}{|c|}{ Conclusión } \\
\hline $\begin{array}{l}\text { Esta es una } \\
\text { investigación } \\
\text { cuantitativa; } \mathrm{La}\end{array}$ & $\begin{array}{c}146 \text { personas } \\
\text { de Tabriz }\end{array}$ & $\begin{array}{l}\text { Tres tipos de } \\
\text { cuestionarios } \\
\text { estándar para }\end{array}$ & $\begin{array}{l}\text { Código de ética } \\
\text { en investigación. }\end{array}$ & $\begin{array}{l}\text { los result } \\
\text { investiga } \\
\text { relación s }\end{array}$ & $\begin{array}{l}\text { los de esta } \\
\text { ón, existe una } \\
\text { snificativa positiva }\end{array}$ & \multicolumn{2}{|c|}{$\begin{array}{l}\text { El investigador concluye que, } \\
\text { los resultados también indican } \\
\text { que existe una relación positiva }\end{array}$} \\
\hline
\end{tabular}

Ciencia Latina Revista Científica Multidisciplinar, Ciudad de México, México.

ISSN 2707-2207 / ISSN 2707-2215 (en línea), julio-diciembre, 2020, Volumen 4, Número 2.

https://doi.org/10.37811/cl_rcm.v4i2.133 p. 966 


\begin{tabular}{|c|c|c|c|c|}
\hline $\begin{array}{l}\text { información sobre la } \\
\text { revisión de la literatura } \\
\text { se recopila a través del } \\
\text { método bibliográfico. } \\
\text { y estudio de } \\
\text { investigaciones } \\
\text { relacionadas } \\
\text { Coeficiente de } \\
\text { correlación de Pearson y } \\
\text { Se han utilizado } \\
\text { métodos de regresión } \\
\text { múltiple para analizar } \\
\text { los resultados } \\
\text { estadísticos. }\end{array}$ & $\begin{array}{l}\text { El municipio } \\
\text { ha participado } \\
\text { en esta } \\
\text { investigación } \\
\text { como muestra } \\
\text { estadística. }\end{array}$ & $\begin{array}{c}\text { reunir los datos } \\
\text { necesarios sobre } \\
\text { estructura } \\
\text { organizacional, } \\
\text { creatividad y } \\
\text { compromiso. El } \\
\text { cuestionario de } \\
\text { Robin se usa para } \\
\text { reunir } \\
\text { información } \\
\text { necesaria sobre la } \\
\text { dimensión de la } \\
\text { estructura } \\
\text { organizacional y } \\
\text { para información } \\
\text { relacionada con la } \\
\text { organización }\end{array}$ & $\begin{array}{l}\text { entre los elementos de la } \\
\text { estructura organizacional y la } \\
\text { creatividad organizacional, y } \\
\text { todos los elementos de la } \\
\text { estructura organizacional } \\
\text { tienen un efecto positivo en la } \\
\text { creatividad organizacional. }\end{array}$ & $\begin{array}{l}\text { significativa entre la } \\
\text { creatividad organizacional y el } \\
\text { Compromiso organizacional }\end{array}$ \\
\hline
\end{tabular}




\section{Discusión}

Posterior a la revisión sistemática de los artículos, del 100\%, el 30\% se encuentra en el continente de Asia integrados por los países de Irán, Palestina y Jordania. Asimismo, el $50 \%$ corresponden al continente europeo integrado por artículos de Holanda, Bosnia, Herzegovina, España, Alemania y Suecia, finalmente el 20\% se encuentra los países del continente de África, integrado por Zimbabwe y Sudáfrica.

Asimismo, Al-Bataineh et al., (2019), en su estudio realizado en la municipalidad de Jordania, indica que es muy importante la labor de guiar a los responsables políticos, a través de la motivación y así como el empoderamiento para mejorar el compromiso organizacional en las instituciones en un país que está en desarrollo.

Por otro lado, Kirst \& Lang, (2019), en la investigación realizada en las municipalidades de Lüneburg, Alemania menciona que la sostenibilidad aplicadas en los gobiernos locales apoyan al desarrollo local fortaleciendo la organizacionalmente la institución.

Como también, de Gier, (2018), realizo una investigación en las municipalidades de Alemania, enfocado a mejorar las organizaciones a través del uso de las tecnología de información indicando que con la adopción de las mismas se ve una mejora en los niveles superiores así también en las autoridades.

De la misma manera, Nyström et al., (2018), en la investigación realizada en las municipalidades de Suecia, donde se implementa trabajar de manera más sistemática con el mapeo, el análisis y el establecimiento de objetivos, lo que permitió un mejoramiento organizacional de las instituciones en sus gestión.

Igualmente, Díaz-Villavicencio et al., (2017), en la instigación realizada en gobiernos locales de España, menciona la importancia de uso de eco eficiencia, para mejorar el uso de los recursos además de que los gobiernos locales no cuentan con muchos recursos, por lo cual su so fortalece la estructura organizacional

Como también, Manuere et al., (2017), e la investigación realizada en los municipios de Zimbabwe, África, menciona la importancia del entrenamiento y el desarrollo de la satisfacción laboral, que permita mejorara la gestión municipal.

Del mismo modo, Jašarević et al., (2017), en su estudio realizado a las municipalidades de en Bosnia y Herzegovina, menciona que los problemas y aspectos que suceden se resuelven de cara con los ciudadanos, indican la existencia de desviaciones, 
comportamiento, falta de reglas de conducta, disfunción organizacional y pobre motivación de los empleados.

En el mismo enfoque, Salem Deeb et al., (2016), en la investigación realizada en la municipalidad de Gaza, plantea la gestión de conocimiento como una herramienta potente para el fortalecimiento de las instituciones que incide en la gestión.

Asimismo, Dadashi Jokandan et al., (2015), también hace hincapié en el uso de las TIC, con la finalidad de mejorar los procesos en la estructura organizacional.

Finalmente, Holagh et al., (2014), menciona a la importancia de la creatividad organizacional en el estudio realizado en los municipios de Irán, donde busca evidenciar la importancia del compromiso organizacional.

Por los cual podemos inferir que el desarrollo organizacional tiene un impacto de mejora en las municipalidades, siempre que estos partan del cambio internos de las personas a través del uso de las herramientas, como pueden ser las capacitaciones o también por así decirlo el uso de las TIC y la eco eficiencia, permitiendo mejorara la gestión de las municipalidades.

\section{CONCLUSIÓN O CONSIDERACIONES FINALES}

La evaluación de los 10 artículos científicos, enfocados al desarrollo organizacional para mejorar la gestión municipal pertenecen al tipo y diseño de estudios descriptivos. El 50\% de los artículos científicos se encuentran enfocados a desarrollo de competencias y habilidades de los trabajadores municipales a través de capacitaciones, 30\% se encuentra enfocado al uso de la TIC, para lograr la gestión y $20 \%$ menciona el uso de la coeficiencia y metodologías aplicadas a al mapeo de objetivos institucionales.

Tomando en cuenta los artículos podemos mencionar que la gestión de las municipalidades son deficientes en Asia, Europa y África, por ello las autoridades y directivos de los municipios deben tomar en cuenta diferentes metodologías para el desarrollo organizacional, además para el desarrollo de futuras investigaciones tomar otras variables y dimensiones de gestión que permitan escudriñar con mayor énfasis en la problemática que aquejan los gobiernos locales.

\section{LISTA DE REFERENCIAS}

Al-Bataineh, O. H., Ibrahim, R. B. M., \& Fadzil, A. F. M. (2019). The Effects of Motivation, Empowerment and Organizational culture on Organizational Commitment of Municipalities in Jordan. International Journal of Academic Research in Business and Social Sciences, 9(9), 101-117. 
https://doi.org/10.6007/IJARBSS/v9-i9/6272

Dadashi Jokandan, A., Fattahi, M., \& Ghane, S. F. (2015). Examining the Role of Information and Communication Technology in the Organizational Structure of the QaemShahr Municipality.

de Gier, J. (2018). IT Governance of Dutch Municipalities and Digital Information Management. New Review of Information Networking, 23(1-2), 36-46. https://doi.org/10.1080/13614576.2018.1537798

Díaz-Villavicencio, G., Didonet, S. R., \& Dodd, A. (2017). Influencing factors of ecoefficient urban waste management: Evidence from Spanish municipalities. Journal of Cleaner Production, 164, 1486-1496. https://doi.org/10.1016/j.jclepro.2017.07.064

Holagh, S. R., Noubar, H. B. K., \& Bahador, B. V. (2014). The Effect of Organizational Structure on Organizational Creativity and Commitment within the Iranian Municipalities. Procedia - Social and Behavioral Sciences, 156, 213-215. https://doi.org/10.1016/j.sbspro.2014.11.175

Jašarević, S., Miličević, D., Brdarević, S., \& Lemeš, S. (2017). Measurement of organizational culture in public administration: Case study of 5 municipalities in Bosnia and Herzegovina. International Journal for Quality Research, 11(3), 655676. https://doi.org/10.18421/IJQR11.03-11

Kirst, E., \& Lang, D. (2019). Perspectives on Comprehensive Sustainability-Orientation in Municipalities: Structuring Existing Approaches. Sustainability, 11(4), 1040. https://doi.org/10.3390/su11041040

Latinobarometro. (2017). Latinobarómetro Database. http://www.latinobarometro.org/latContents.jsp

Manuere, F., Manuere, \& Faitira. (2017). Human Capital Development Programmes and their Effect on the Job Satisfaction of Workers in Zimbabwe Urban Municipalities: The Case of Chinhoyi. International Journal of Academic Research in Business and Social Sciences, 7(12), 874-889.

Miranda Chuquimango, L. M. (2016). Programa de capacitación para mejorar el desempeño laboral del personal administrativo de transportes Rodrigo Carranza S.A.C - Trujillo: 2016. Universidad Nacional de Trujillo. http://dspace.unitru.edu.pe/handle/UNITRU/8042

Muñoz Saravia, A. (2007). Los Metodos Cuantitativo y Cualitativo en la Evaluacion de Impactos en Proyectos de Inversion Social. http://www.postgradoune.edu.pe/pdf/documentos-academicos/evaluacion-de-laeducacion-superior/2.pdf

Nyström, M. E., Höög, E., Garvare, R., Andersson Bäck, M., Terris, D. D., \& Hansson, J. (2018). Exploring the potential of a multi-level approach to improve capability for continuous organizational improvement and learning in a Swedish healthcare region. BMC Health Services Research, 18(1). https://doi.org/10.1186/s12913-018-3129-3 
Salem Deeb, B. R., El-Shorafa, Y. A., \& Al-Habil, W. A. (2016). The Effect of Some Organizational Factors on the Knowledge Management Implementation at the Municipality of Gaza. IUG Journal of Economics and Business Studies, 24(1), 116. https://doi.org/10.12816/0025731

Tirado, K. (2014). Gestión administrativa y la calidad de servicio al cliente, en el Colegio Químico Farmacéutico de La Libertad - Trujillo. (Tesis de pregrado) Universidad Nacional de Trujillo. Trujillo. Perú.

Urbina Nájera, M. C. (2017). Liderazgo global efectivo : ¿Cuáles son las habilidades esenciales para un liderazgo efectivo en equipos multiculturales? $/$. Http://Biblioteca.Utb.Edu.Co/Notas/Tesis/0074445.Pdf.

https://repositorio.utb.edu.co/handle/20.500.12585/1246\#.Xs_0rV913zI.mendeley 